Journal of Computer Science 6 (1): 67-74, 2010

ISSN 1549-3636

(C) 2010 Science Publications

\title{
Feature Extraction for Characterization of Breast Lesions in Ultrasound Echography and Elastography
}

\author{
${ }^{1}$ Shirley Selvan, ${ }^{1}$ M. Kavitha, ${ }^{1}$ S. Shenbagadevi and ${ }^{2}$ S. Suresh \\ ${ }^{1}$ Center for Medical Electronics, Department of Electronics and Communication Engineering, \\ College of Engineering, Anna University, Chennai 600025, India \\ ${ }^{2}$ Department of fetal medicine, Mediscan Systems, Chennai 600014, India
}

\begin{abstract}
Problem statement: Elastography is developed as a quantitative approach to imaging linear elastic properties of tissues to detect suspicious tumors. We propose an automatic feature extraction method in ultrasound elastography and echography for characterization of breast lesions. Approach: The proposed algorithm was tested on 40 pairs of biopsy proven ultrasound elastography and echography images of which 11 are cystic, 16 benign and 13 malignant lesions. Ultrasound elastography and echography images of breast tissue are acquired using Siemens (Acuston Antares) ultrasound scanner with a 7.3 MHz linear array transducer. The images were preprocessed and subjected to automatic threshold, resulting in binary images. The contours of a breast tumor from both echographic and elastographic images were segmented using level set method. Initially, six texture features of segmented lesions are computed from the two image types followed by computing three strain and two shape features using parameters from segmented lesions of both elastographic and echographic images. Results: These features were computed to assess their effectiveness at distinguishing benign, malignant and cystic lesions. It was found that the texture features extracted from benign and cystic lesions of an elastogram are more distinct than that of an ultrasound image. The strain and shape features of malignant lesions are distinct from that of benign lesions, but these features do not show much variation between benign and cystic lesions. Conclusion: As strain, shape and texture features are distinct for benign, malignant and cystic lesions, classification of breast lesions using these features is under implementation.
\end{abstract}

Key words: Elastography, ultrasound, breast lesion, elastogram, strain

\section{INTRODUCTION}

Non-invasive methods used to diagnose breast cancer still have limitations. Detection techniques are currently based primarily on physical examination, mammography and sonography. Mammography and sonography are currently the most sensitive noninvasive modalities for detecting breast cancer. Ultrasonography is chosen as the work up tool. Ultrasound has long been used to distinguish between harmless, fluid-filled cysts and solid masses. However, solid masses are not always malignant. For example, both fibroadenomas and scirrhous carcinomas are solid and stiff, but only the latter are malignant. The sonographic features for benign and malignant lesions have been shown to override each other substantially (Stavros et al., 1995). In mammography, the compression of breast tissue and the repositioning of the breast for different views cause patient discomfort. It is also difficult to image dense breast tissue in mammography. These limitations of mammography and sonography and the need, not to miss a malignant lesion in the early stage of disease leads to invasive surgical biopsy that cause unnecessary patient discomfort, anxiety and hospitalization in addition to increasing costs to the patient. This substantial problem remains in breast cancer diagnosis.

Existing imaging technologies have limited detection capabilities, as well as an inability to differentiate benign from malignant cancers. Overall, there is a need for alternative non-invasive methods of detection and diagnosis of breast lesions with greater sensitivity and specificity.

Mechanical measurements have shown that pathological tissue can be up to 30 times stiffer than normal tissue (Parkin et al., 2005). Ultrasound

Corresponding Author: Shirley Selvan, Center for Medical Electronics,

Department of Electronics and Communication Engineering, College of Engineering,

Anna University, Chennai 600025, India 
elastography described by Ophir et al. (1991) is a method for measuring the stiffness/elastic properties of tissues (Stavros et al., 1995; Parkin et al., 2005; Ophir et al., 1991; Hall et al., 2003). The basic operating principle of elastography involves the comparison of the spatial arrangement of tissue before and after compression. This scanning modality which can provide information about stiffness of the lesions is currently used for detecting and identifying lesions in the breast. Paired images are obtained, consisting of the standard B-mode image on the left and a pure strain image on the right (Hall et al., 2003).

Benign lesions usually appear smaller or of the same size on sonograms as well as on elastograms. In case of malignant lesions, the size appears larger on the elastogram (Elisa et al., 2000). Unlike benign lesions that have smoother borders and are more loosely bound to the adjacent perilesional tissue, thereby being more mobile, malignant tumors are known to form stellate boundaries that become firmly bound to the surrounding tissue through infiltration. During an applied compression, benign lesions tend to undergo motion in an opposite direction to that of the compression. On the other hand, malignant lesions move in the direction of compression while pulling the perilesional tissue in the same direction; thereby causing the perilesional tissue also to appear stiffer on the elastogram as well.

Studies have demonstrated that B-mode ultrasound imaging tends to underestimate the size of a tumor compared to pathology measurement. It is shown that the size of a breast tumor is larger in elasticity images than in B-mode ultrasound images and it is a reasonable hypothesis that the tumor size in elasticity images is a more accurate representation of that measured at pathology.

Because of the ease with which compression can be applied to the breast, it is an ideal organ on which to perform elastography. Due to the relative stiffness of breast cancer tissue, as compared to benign fibro adenomas and cysts, a method such as elastography which estimates tissue stiffness may be an attractive tool for distinguishing benign from malignant lesions.

In the diagnosis of breast cancer using elastography, Garra et al. (1997); Hall et al. (2003) proposed several diagnostic criteria such as lesion visualization, relative brightness and margin irregularity by capturing the radiofrequency data of the reflected echoes after giving compression to the lesion. Instead of using radiofrequency data, Steinberg et al. (2001) and Moon et al. (2005) used continuous ultrasound images which are obtained through probe compression.
Automated detection of tumor margin in breast elastography is desired for diagnostic purposes. $\mathrm{Wu}$ Liu et al. (2007) proposed a preliminary segmentation algorithm using the coarse to fine active contour method. This method is effective for segmenting regions of images that have a relatively regular, well circumscribed single margin but might not be effective in segmenting speculated masses with irregular margins as seen in malignant tissue.

Xia and Liu (2007) method refers to contour evolution which improvises on the coarse to fine active contour method proposed by Liu et al. (2007). This method can handle features that the active contour method has difficulties with, including self intersecting contours and changes in topology.

In present study, ultrasound images of the breast, namely the B mode ultrasonogram and elastogram are preprocessed by a Speckle Reducing Anisotropic Diffusion filter ( $\mathrm{Yu}$ and Acton, 2002). An automatic threshold applied to the preprocessed images results in binary images. The lesion region is identified by Renbo Xia's method of contour evolution. Three sets of features namely texture, strain and shape features are computed from the segmented lesions of both image types.

The research is organized as follows. The contours of a breast tumor are evolved from the acquired ultrasound elastography and echography images of breast, followed by segmentation of breast lesions. Texture, strain and shape features are extracted from the segmented lesions.

\section{MATERIALS AND METHODS}

Image acquisition: Ultrasound elastography and echography images of breast are acquired using Siemens (Acuston Antares) ultrasound scanner with a 7.3 MHz linear array transducer. Initially $\mathrm{B}$ mode image of the lesion is taken, following which a slight compression is applied. The effect of breathing and heart beat produce the required compression. The elastogram is generated by the machine by comparing pre and post compressed RF signals and the elastogram is displayed adjacent to the $\mathrm{B}$ mode image. The ultrasonograms and elastograms generated are gray scale images.

Segmenting the lesion: Due to noise and speckles in the ultrasound B mode and elastographic images, noise filtering and edge-enhancement are required. There are several fundamental requirements of noise filtering methods for medical images. One, it should not lose the important information of object boundaries and detailed 
structures. Two, it should efficiently remove noise in the homogeneous regions and finally, it should enhance morphological definition by sharpening discontinuities. The Speckle Reducing Anisotropic Diffusion (SRAD) filter (Yongjian Yu and T. Scott Acton, 2002) meets these requirements of noise filters and also improves the image quality significantly while preserving the important boundary information and hence, in present study, speckle reducing anisotropic diffusion filtering of real elastography and ultrasound B mode images is done to reduce noise and speckles.

Segmentation is required to separate the tumor region from its background. Segmentation algorithms for grey scale images are based on one of the two basic properties of image intensity values: discontinuity and similarity. In the first category, the approach is to partition the image based on abrupt changes in the intensity, such as edges in an image. The principal approaches in the second category are based on partitioning an image into two regions that are similar according to a set of predefined criteria. In present study, automatic threshold and level set active contour method, based on the above criteria are used for segmentation.

An automatic threshold-determination method, proposed by Otsu (1997), can choose the threshold to minimize the intraclass variance of the black and white pixels automatically. An additional control scheme is allowed to enable the user to change the threshold value when he is not satisfied with the threshold value assigned by this automatic method. In an elastogram, the tumor region appears to be darker and the background bright. In present study, the preprocessed images are subjected to the above mentioned automatic threshold scheme, resulting in binary images as this aids in separating the lesion from its background. The area of lesion is segmented from the binary image by applying level set segmentation technique.

Level set method: Level sets are first described by Osher and Sethian (1988) as a method for capturing moving fronts. In the level set formulation, the segmentation problem is equivalent to the computation of a surface $\Gamma(\mathrm{t})$ that propagates in time along its normal direction. The $\Gamma$ surface is also called a propagating front, which, according to Osher and Sethian (1988), is embedded as a zero level of a timevarying higher dimensional function $\phi(\mathrm{x}, \mathrm{t})$ :

$\Gamma(\mathrm{t})=\left\{\mathrm{x} \in \mathfrak{R}^{3} \mid \phi(\mathrm{x}, \mathrm{t})=0\right\}$

An evolution equation for an interface $\Gamma$, where $\Gamma$ is a closed curve in $\mathfrak{R}^{2}$, can be written in a general form: $\frac{\partial \phi}{\partial \mathrm{t}}+\mathrm{F}|\Lambda \phi|=0$

The function $\phi$ describes a curve defined by:

$$
\phi(x, t)=d
$$

where, $\mathrm{d}$ is a signed distance between $\mathrm{x}$ and the surface $\Gamma$. If $\mathrm{x}$ is inside (resp. outside) of $\Gamma$, then $\mathrm{d}$ is negative (resp. positive). The function $\mathrm{F}$ is a scalar speed function that depends on image data and the function $\phi$. The main drawback of this procedure is that during the evolution, $\phi$ can assume sharp or flat shapes. To overcome this problem, $\phi$ is initialized as a signed distance function before the evolution. Later, during the evolution, it is periodically reshaped to be a signed distance function (Li et al., 2005). Li et al. (2005) proposed a modification of traditional variational level sets to overcome the problem of the reshaping of function $\phi$ to be a distance function within the evolution cycle.

In present study, variational level sets are used, which are more robust than those originally proposed by Osher and Sethian because they incorporate shape and region information into the level set energy functions. Here, the initial contours of lesions of both ultrasound and elastography images are determined by the method proposed by Xia and Liu (2007). This algorithm consists of finding all endpoints in an edge map. All the valid pairs are established. The linking cost for all the valid pairs is computed. All the pairs keyed on cost are placed in a heap with the minimum cost pair at the top. The pair of least cost from the heap is iteratively linked and the connected pair is removed. This algorithm has been applied to both ultrasound B mode and elastography images. The segmentation results are shown in Fig. 1-4.

After tumor contours are segmented from the elastographic and US B mode images, six texture features, three values for features of strain-contour difference, perimeter difference and area difference and two values for the shape feature- solidity and width to height difference are computed.

Feature extraction: Features are to be computed from the segmented region to identify lesions into one of the three types, namely benign, malignant or cystic.

Strain and shape features: The size of a malignant breast tumor is larger in elasticity images than shown in B-mode ultrasound images. Similarly, the size of a benign lesion in elasticity images is smaller or equal to B-mode ultrasound images. Based on the difference in size of lesions in elasticity images and B-mode ultrasound images it is decided to concentrate on features namely area difference, perimeter difference and contour difference (Kavitha et al., 2009). 


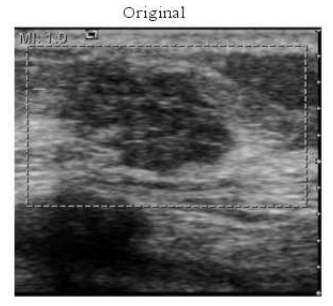

Fig. 1a: Original US B mode image of malignant lesion

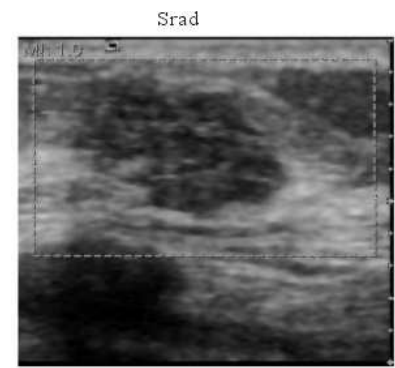

Fig. 1b: Filtered image

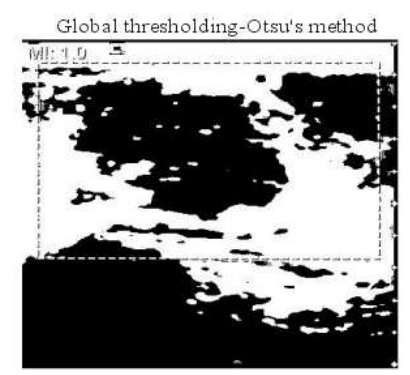

Fig. 1c: Image after applying automatic threshold

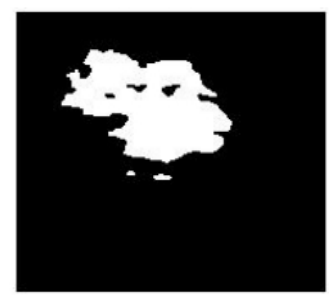

Fig. 1d: Final contour

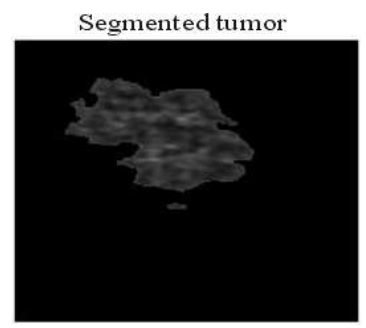

Fig. 1e: Segmented tumor (ROI)

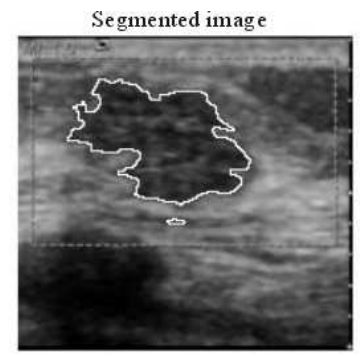

Fig. 1f: Computer delineated margin of lesion

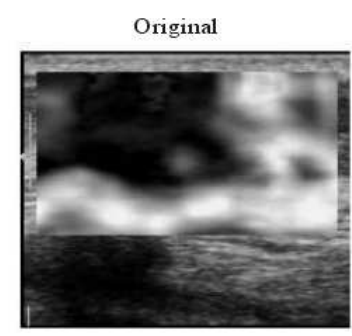

Fig. 2a: Original elastogram of malignant lesion

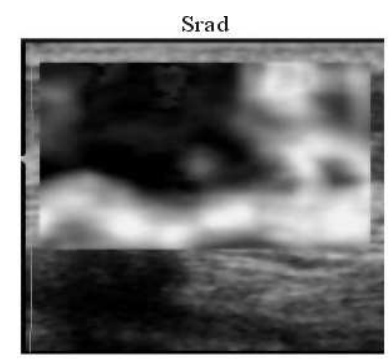

Fig. 2b: Filtered image



Fig. 2c: Image after applying automatic threshold

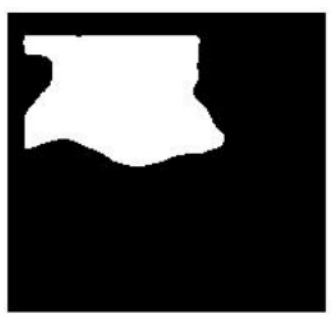

Fig. 2d: Final contour 


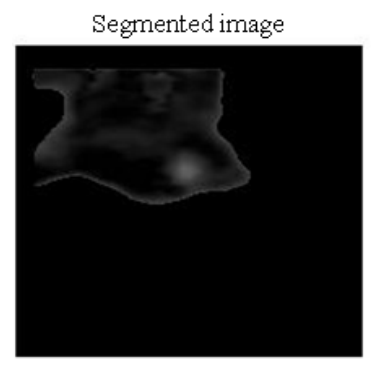

Fig. 2e: Segmented tumor (ROI)

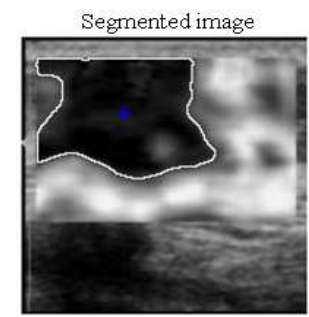

Fig. 2f: Computer delineated margin of lesion

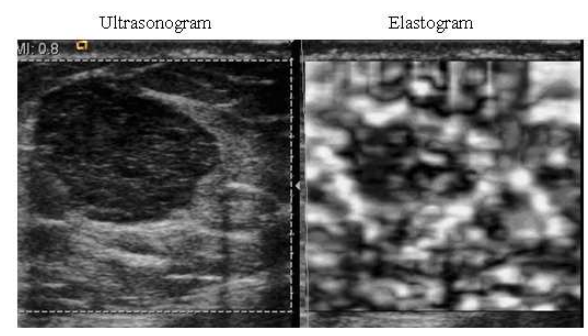

Fig. 3a: Ultrasound image and elastogram of a benign lesion

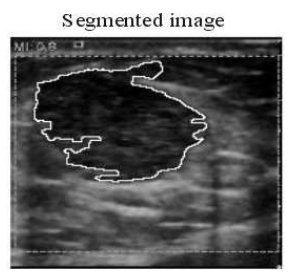

Fig. 3b: Computer delineated margin of lesion of ultrasonogram

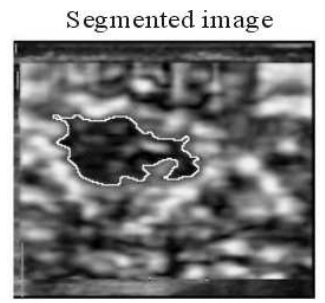

Fig. 3c: Computer delineated margin of lesion of elastogram

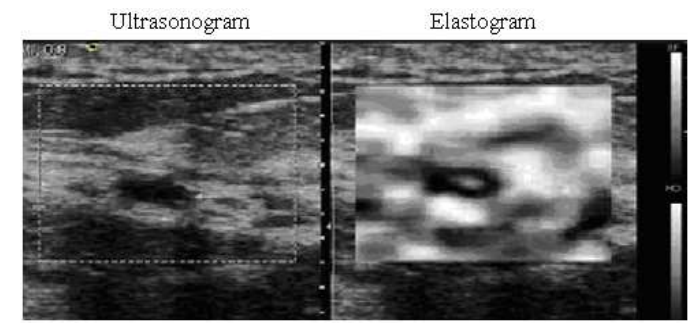

Fig. 4a: Ultrasound image and elastogram of a cystic lesion

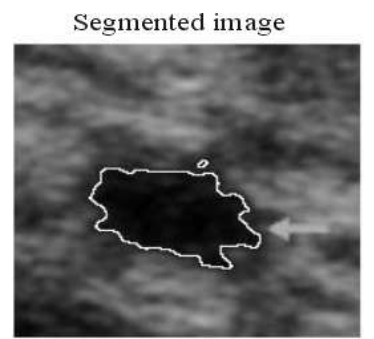

Fig. 4b: Computer delineated margin of lesion of ultrasound image a sound image

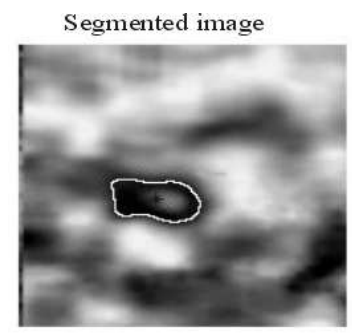

Fig. 4c: Computer delineated margin of lesion of elastogram

Area difference: The area difference is used to compare areas of lesions between two images, as lesion area changes in accordance to the applied pressure. The area difference is defined as the difference between areas of lesions in the ultrasound images and elastograms divided by the number of pixels in the lesion region of the ultrasound image:

$$
\text { Area difference }=\frac{a_{u}-a_{e}}{a_{u}} \times 100
$$

where, $a_{u}$ and $a_{e}$ are the lesion areas of ultrasonogram and elastogram respectively.

Perimeter difference: The perimeter of the lesion is computed by calculating the distance between each adjoining pair of pixels around the border of the region. It is length of the nuclear envelope calculated as the 
length of a polygonal approximation of the boundary (B), where $\mathrm{p}$ is perimeter of lesion. Polygonal approximation is approximating a closed curve as a $2 \mathrm{D}$ polygon by which a simple representation of the planar object boundary is provided:

$$
\mathrm{p}=\sum_{\mathrm{x} \in \mathrm{B}} 1
$$

The perimeters of lesions in ultrasonogram and elastogram are computed.

Solidity: Shape values can be used to distinguish between benign and malignant tumors. Benign lesions usually have smooth shapes and so they produce a regular shape in both ultrasound and elastographic images whereas malignant lesions present irregular shapes in elastograms. This difference can be obtained in terms of a feature called solidity:

$$
\text { Solidity }=\frac{\mathrm{cvX}_{\mathrm{ar}}}{\sum \frac{\mathrm{cVX}_{\mathrm{ur}}-\text { tumor }_{\mathrm{ar}}}{\mathrm{N}_{\mathrm{tot}}}}
$$

Where:

$\mathrm{N}_{\text {tot }} \quad=$ The total number of imaging modalities involved

$\mathrm{cvx}_{\mathrm{ar}}=$ The area obtained from the convex hull of a tumor

tumor $_{\mathrm{ar}}=$ The area of tumor

The convex hull (Zunic and Rosin, 2002) is the smallest convex set containing a tumor and resembles a rubber band wrapped around the tumor.

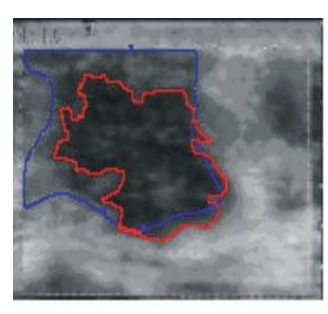

(a)

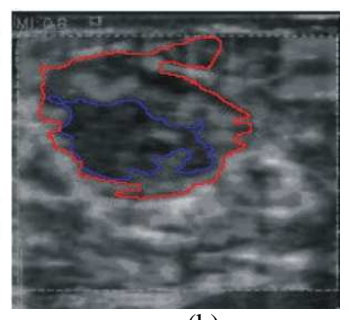

(b)

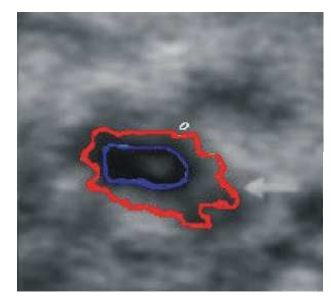

- Contour of the lesion in
an elastogram
- Contour of the lesion in
an ultrasonogram

(c)

Fig. 5: Registered contour. (a) malignant lesion; (b) benign lesion; (c) cystic lesion
Contour difference: The contour difference feature is used to compare differences of contours between the ultrasound $\mathrm{B}$ mode lesion and elastogram lesion. Initially the contours are registered by linear conformal method transformation. It is the transformation of an image by scaling the image, rotating the image and translating the image to a different coordinate system. Intensity weighted centroids are used as reference points for the two lesions (Moon and Chang, 2005):

Contour difference $=\frac{\mathrm{N}_{\text {condiff }}}{\mathrm{N}} \times 100$

Where:

$\mathrm{N}_{\text {condif }}=$ The pixel difference between the two registered contours

$\mathrm{N}=$ The number of tumor pixels in the ultrasound image (Moon and Chang, 2005)

The registered contours are shown in Fig. 5a-c for malignant, benign and cystic lesions respectively.

Width to height difference: Due to applied stress, a benign lesion appears smaller in an elastogram compared to B-scan image, whereas a malignant lesion appears larger in an elastogram because of its solid nature. Based on this, the width to height difference can be taken as a feature. An ellipse that has same normalized second central moment as the region of interest is drawn. The major axis length (height) and minor axis length (width) of the ellipse are computed. The ratio of width to height is calculated for lesions of ultrasonogram and elastogram respectively.

Texture features: The texture features represent changes of grey level intensity. The second order statistical features namely standard deviation, energy, entropy, dissimilarity, homogeneity and contrast are computed using Grey level Co-occurrence Matrix (Haralick et al., 2007) from ultrasonogram and elastogram. This square matrix estimates the inter-pixel positioning and each cell carries the count of the number of times a pixel pair occurs as a function of two other parameters, the distance ' $\mathrm{d}$ ' and the angle ' $\theta$ ' between them. Generally the value of ' $d$ ' is fixed at 1 and $\theta$ is allowed to vary in steps of $45^{\circ}$ clockwise up to $180^{\circ}$ to get 4 such matrices completely describing the image region. The features are obtained from 20 ultrasound images and 20 elastograms. The minimum and maximum value of each feature is shown in Table 1-3. The obtained features are presented in Table 1-3. 
Table 1: Texture features of ultrasonogram

\begin{tabular}{llll}
\hline & (Minimum to maximum) & \\
Features & Benign lesion & Malignant tumor & Cystic lesion \\
\hline Energy & $10000-50000$ & $500-10000$ & $7000-43000$ \\
Entropy & -830 to -1290 & -120 to -280 & -2000 to -16000 \\
Dissimilarity & $100-500$ & $40-10000$ & $3000-15000$ \\
Homogeneity & $30-430$ & $57-78$ & $300-2000$ \\
Contrast & 100 to 1000 & $150-3000$ & $450-20000$ \\
SD & $20-60$ & $0.22-1.0$ & $0.6-35$ \\
\hline
\end{tabular}

Table 2: Texture features of elastogram

(Minimum to maximum)

\begin{tabular}{llll} 
& & & \\
Features & Benign lesion & Malignant tumor & Cystic lesion \\
\hline Energy & $300-1000$ & $2000-8000$ & $3000-20000$ \\
Entropy & -60 to -540 & -300 to -500 & -780 to -8000 \\
Dissimilarity & $3000-4000$ & $80-1200$ & $1300-20000$ \\
Homogeneity & $52-278$ & $268-530$ & $160-1000$ \\
Contrast & 100 to1000 & $90-2500$ & $430-3000$ \\
SD & $0.1-0.25$ & $0.7-40$ & $0.39-0.84$ \\
\hline
\end{tabular}

Table 3: Strain and shape features from ultrasongram and elastogram (Minimum to maximum)

\begin{tabular}{lll} 
& ------------------------------------ \\
Features & Benign and cystic lesion & Malignant tumor \\
\hline Area difference & $5-100$ & -60 to -10000 \\
Solidity & $0.6-0.8$ & $0.2-0.6$ \\
Perimeter difference & $10-100$ & $100-1000$ \\
Contour difference & $7-100$ & $1-10$ \\
Width-height ratio & -40 to -150 & $15-100$ \\
\hline
\end{tabular}

\section{RESULTS}

The segmentation results are shown in Fig. 1-4. Figure 1a-f show the various stages of filtering and segmentation of an US B mode image of a malignant lesion. Figure $2 \mathrm{a}-\mathrm{f}$ show the various stages of filtering and segmentation of an elastogram of a malignant lesion. Figure $3 \mathrm{a}-\mathrm{c}$ shows the ultrasound image and elastogram of a benign lesion and their segmented images. Figure $4 a-c$ shows the ultrasound image and elastogram of a cystic lesion and their segmented images. The computed delineated margin is the white outline.

The features extracted are listed in Table 1-3. Table 1 presents the texture features of malignant, benign and cystic lesions of an US B mode image. Table 2 presents the texture features of malignant, benign and cystic lesions of an elastogram. Table 3 presents the strain and shape features obtained from parameters of both US and elastography images.

\section{DISCUSSION}

The above algorithm is tested on 40 pairs of ultrasound and elastography biopsy proven images where 11 are cystic, 16 benign and the remaining 13 , malignant tumors. Malignant masses are stiffer and therefore deform less than benign masses, besides they appear darker and larger than benign masses on an elastogram. A benign tumor would be of comparable size in both sonogram and elastogram. A cyst is characterized by its inner anechogenic substance and thin echogenic outer wall nature. It is depicted as nidus (bull's eye appearance) in an elastogram (Fig 4c). The computed delineated margin is the white outline.

Texture features of a lesion in an elastogram provide better information about the inner details of cyst than texture features of a lesion in an US B mode image. Hence a cyst is well identified in an elastogram than in an US B mode image. From Table 2, it is seen that there is not much of difference between the elastogram texture features of benign and malignant lesions, but there is much difference between the elastogram texture features of benign and cystic lesions.

From Table 3, it is observed that strain and shape features extracted using parameters from US B mode image and elastogram, well differentiate the malignant tumors from benign and cystic lesions.

Hence, elastogram texture features are superior in differentiating cystic lesions from benign conditions. The strain and shape features well differentiate the benign from malignant lesions. Hence, we conclude that it is appropriate to combine the information obtained from both US elastography and US B mode images for better diagnosis.

\section{CONCLUSION}

In this proposed method the two sets of images are initially preprocessed by anisotropic diffusion filtering and then by an automatic threshold technique. The level set method is utilized to segment the lesion in the combined image. The texture, strain and shape features are computed from the segmented lesions. Some of the features are distinct in an elastogram for the three specified conditions and hence elastogram increases the specificity of diagnosis. Classification of breast lesions using texture and strain features obtained from ultrasound images and elastograms is under implementation.

\section{REFERENCES}

Elisa, E., Konofagou, Tim Harrigan and Jonathan Ophir, 2000. Shear strain estimation and lesion mobility assessment in elastography. Ultrasonics, 38: 400-404. DOI: 10.1016/S0041-624X (99)00125-0 
Garra, B.S., E.I. Cespedes, J. Ophir, S.R. Spratt and R.A. Zuurbier et al., 1997. Elastography of breast lesions: Initial clinical results. Radiology, 202: 79-86.

Hall, T.J., Y. Zhu and C.S. Spalding, 2003. In vivo realtime freehand palpation imaging. Ultrasound Med. Biol., 29: 427-435. PMID: 12706194

Haralick, R., K. Shanmugam and I. Dinstein, 1973. Texture features for image classification. IEEE Trans. Syst. Man Cybernetics, 3: 610-621. DOI: 10.1109/TSMC.1973.4309314

Kavitha, M., S. Shirley Selvan, Shenbagadevi and S. Suresh, 2009. Automatic segmentation and feature extraction of breast lesions. Proceedings of the International Conference on Biology Medical Informatics and Signal Processing, Mar. 12-14, India, pp: 50.

Li, C., C. Xu, C. Gui and M. Fox, 2005. Level set evolution without re-initialization: A new variational formulation. Proceedings of the IEEE Conference on Computer Vision and Pattern Recognition, June 20-25, IEEE Xplore Press, San Diego, USA., pp: 430-436. DOI: 10.1109/CVPR.2005.213

Moon, W.K. and R.F. Chang, 2005. Solid breast masses: Classification with Computer-aided analysis of continuous US images obtained with probe compression. Radiology, 236: 458-464. PMID: 16040902

Ophir, J., I. Cespedes, H. Ponnekanti, Y. Yazdi and X. Li, 1991. Elastography: A quantitative method for imaging the elasticity of biological tissues. Ultrasound Imag., 13: 111-134. DOI: 10.1109/IEMBS.2007.4352227

Osher, S. and J.A. Sethian, 1988. Fronts propagating with curvature-dependent s peed: Algorithms based on Hamilton-Jacobi formulations. J. Computat. Phys, 79: 12-49.

Otsu, N., 1997. A threshold selection method form gray-level histograms. IEEE Trans. Syst. Man Cybern., $\quad 9$ 62-66. http://www.citeulike.org/group/1650/article/11169 82
Parkin, D.M., F. Bray, J. Ferlay and P. Pisani, 2005. Global cancer statistics. CA Cancer J. Clin., 55: 74-108. PMID: 15761078

Renbo, Xia and Weijun Liu et al., 2007. An optimal initialization technique for improving the segmentation performance of Chan-Vese model. Proceedings of the IEEE International Conference on Automation and Logistics, Aug. 18-21, IEEE Xplore Press, Jinan, pp: 411-415. DOI: 10.1109/ICPR.2006.713

Stavros, A.T., D. Thickman, C.L. Rapp, M.A. Dennis and S.H. Parker et al.,1995. Solid breast nodules: Use of sonography to distinguish between benign and malignant lesions. Radiology, 196: 123-134. http://www.citeulike.org/article-posts/6345923

Steinberg, B.D., D.C. Sullivan and J.A. Birnbaum, 2001. Sonographic discrimination between benign and malignant breast lesions with the use of disparity processing. Acad. Radiol., 8: 704-712. PMID: 11508749

Wu Liu et al., 2007. Segmentation of elastographic images using a coarse-to-fine active contour model. Ultrasound Med. Biol., 32: 397-408. DOI: 10.1016/j.ultrasmedbio.2005.11.011

Yu, Y. and T.S. Acton, 2002. Speckle reducing anisotropic diffusion. IEEE Trans. Image Process., 11: 1260-1270. PMID: 18249696

Zunic, J. and P.L. Rosin, 2002. A convexity measurement for polygons. Proceedings of the British Machine Vision Conference, Sept. 2-5, Cardiff, UK., pp: 173-182. DOI: 10.1109/TPAMI.2004.19 